\title{
Bactericidal activity of oxacillin and glycopeptides against Staphylococcus aureus in patients with endocarditis: Looking for a relationship between tolerance and outcome
}

\author{
Maria Bruna Pasticci ${ }^{*}$, Amedeo Moretti ${ }^{1}$, Giuliano Stagni ${ }^{1}$, Veronica Ravasio ${ }^{2}$, Laura Soavi ${ }^{2}$, Annibale Raglio ${ }^{3}$, \\ Francesca Vailati ${ }^{3}$, Angela Cardaccia ${ }^{1}$, Antonella Santucci ${ }^{1}$, Rita Papili ${ }^{1}$, Alessio Sgrelli ${ }^{1}$, Carlo Pallotto ${ }^{1}$ and \\ Franco Baldelli ${ }^{1}$
}

\begin{abstract}
Background: There is no clear relationship between in vitro bactericidal activity tests and clinical outcome. We studied bactericidal activity of oxacillin, vancomycin and teicoplanin against Staphylococcus aureus isolates in patients with endocarditis and then we sought to determine if there was a relationship between in vitro bactericidal activity and clinical outcome.

Methods: Minimal bacteriostatic and minimal bactericidal concentrations were determined for Staphylococcus aureus strains isolated from patients with endocarditis following standardized methods. Medical records were reviewed retrospectively to collect data on antimicrobial susceptibility at admission, antimicrobial therapy, need for surgery, embolic events and outcome.

Results and Discussion: Sixty-two Staphylococcus aureus strains were studied in 62 patients with endocarditis. Overall, $91.9 \%$ definite, $21 \%$ methicillin resistant and $72.6 \%$ cured. Surgery was performed in $32.3 \%$ and embolic events were documented in $64.5 \%$. Tolerance to oxacillin and teicoplanin was more common than vancomycin tolerance among methicillin susceptible Staphylococcus aureus. Among methicillin resistant Staphylococcus aureus teicoplanin was shown to have a higher rate of tolerance than vancomycin. No statistically significant differences on clinical outcome between oxacillin tolerant and oxacillin non tolerant Staphylococcus aureus infections were observed. Tolerance to oxacillin did not adversely affect clinical outcomes of patients with methicillin susceptible Staphylococcus aureus endocarditis treated with a combination of antimicrobials including oxacillin. The cure rate was significantly lower among patients with methicillin resistant Staphylococcus aureus endocarditis.
\end{abstract}

Conclusions: In vitro bactericidal test results were not valid predictors of clinical outcome. Physicians need to use additional parameters when treating patients with staphylococcal endocarditis.

\section{Background}

In recently published surveys Staphylococcus aureus $(S$. aureus) is reported to overstep viridans Streptococci as a cause of endocarditis (IE) and associated morbidities and mortality $[1,2]$. S.aureus is an extraordinarily adaptable bacterium, developing increasing patterns of resistance

\footnotetext{
* Correspondence: pasticci@unipg.it

'Section of Infectious Diseases, Department of Experimental Medicine and

Biochemical Sciences, University of Perugia, Perugia, Italy

Full list of author information is available at the end of the article
}

which contribute to clinical failures. Penicillin resistance was soon followed by methicillin resistance, which always includes resistance to all beta-lactam antimicrobials and often to several other classes of antibiotics [3]. The efficacy of vancomycin against methicillin resistant S.aureus (MRSA) has been reported to be inferior to that of betalactams against methicillin susceptible S.aureus (MSSA) due to its slower in vitro bactericidal activity with a lower clinical response [4-6]. Recently other reasons for the clinical failure of vancomycin have been indicated and

\section{() Biomed Central}


include: the progressive increase of vancomycin minimum inhibitory concentrations (MICs) over time, but with values still in the susceptibility range, and the emergence of S.aureus showing either glycopeptide heteroresistance, an intermediate level of resistance (GISA) or full resistance (GRSA) [7-10].

Tolerance is another form of antimicrobial resistance of S.aureus hypothesised to be a cause of clinical failure. Tolerance has been described for anti-staphylococcal beta-lactams but involves also glycopeptide antimicrobial agents. There have been studies reporting infections caused by tolerant strains more difficult to eradicate and antimicrobial regimens with bactericidal activity superior to that of bacteriostatic regimens in the treatment of serious S.aureus infections. Tolerant strains are susceptible as judged by MICs but show an increasing resistance to the killing with high minimal bactericidal concentrations (MBCs) and an $\mathrm{MIC} / \mathrm{MBC}$ ratio $\geq 32$. Bactericidal activity can also be evaluated with time killing curves but, independently from the method used, there are theoretical and technical difficulties in performing these tests. Thus, highly standardised methods should be followed. To date, bactericidal activity has been regarded as a desirable characteristic in antimicrobial agents when treating patients with endocarditis, while, routine MBC testing is not recommended because of the technical difficulties associated with these tests [11-15].

The aims of this study were:

a) To determine the in vitro bactericidal activity of oxacillin, vancomycin and teicoplanin against S.aureus isolated in patients with S.aureus endocarditis

b) To look for a relationship between in vitro bactericidal activity and clinical outcome using data available from patients that had been previously enrolled in observational studies on endocarditis.

\section{Methods}

Isolates of S.aureus were collected from patients with IE admitted to the Infectious Disease Departments (IDD) of Perugia and Bergamo from 1988-2009. A total of 30 strains were from Perugia and 32 from Bergamo. The initial blood isolates on the day of admission were stored at $-70^{\circ} \mathrm{C}$ until the time of in vitro testing. MICs and MBCs were determined at the IDD of Perugia under blinded conditions according to CLSI guidelines [16-18].

1) Oxacillin: Cefoxitin disk diffusion using Muller-Hinton agar plates (bioMerieux) and $30 \mu \mathrm{g}$ cefoxitin disk (bio-Merieux) and interpreted according to CLSI break points (16), macro-method MIC using Muller Hinton Broth (MHB), inoculum 1-5 × $10^{5} \mathrm{CFU} / \mathrm{ml}$, stationary phase of growth) (17) and E-test MIC according to the E-test manufacturer (AB Biodisk).
2) Vancomycin: macro-method MIC (MHB, inoculum $1-5 \times 10^{5} \mathrm{CFU} / \mathrm{ml}$, stationary phase of growth) (17) and E-test MIC.

3) Teicoplanin: macro-method MIC (MHB, inoculum 1-5 $\times 10^{5} \mathrm{CFU} / \mathrm{ml}$, stationary phase of growth) (17), E-test MIC.

$\mathrm{MBC}$ was determined by sub-culturing $50 \mu \mathrm{l}$ from each vial without a visible growth onto plates of Muller Hinton agar (MHA), after $24 \mathrm{~h}$ of incubation at $35^{\circ} \mathrm{C}$. The geometric mean of duplicate colony counts were used to determine the $\mathrm{MBC}$ defined as the antibiotic concentration with killing of $99.9 \%$ of the initial inoculum (18). In the presence of Eagle phenomenon, MBC was the antibiotic concentration yielding persistent killing of $99.9 \%$ of the initial inoculum.

$\mathrm{MIC}_{50}$ and $\mathrm{MIC}_{90}$ were determined by interpolation from graphs of cumulated percent strains inhibited versus MIC [19].

MSSA ATCC 29213 was used as a control strain in all these experiments.

Medical records of each patient were reviewed retrospectively to collect data on: demographic data, year of diagnosis, valve localization, hospital or community acquired infection, antimicrobial susceptibility results at the time of patient admission and antimicrobial treatment, surgery, embolic events and outcomes.

There was no research related effect for patients. Patients gave informed consent to be included in the observational protocol on endocarditis cases. The protocol was approved by the institutional ethical committee of Perugia and Bergamo. All activity was conducted in accordance with the Declaration of Helsinki, and national and institutional review board.

Associations among qualitative variables were analysed using the contingency table. The statistical significance was assessed with the Fisher exact test.

\section{Results}

Sixty-two isolates of S.aureus were collected from patients with endocarditis, 58\% male, 91.9\% definite (DE) and $8.1 \%$ possible (PE) according to Duke criteria, $47(75.8 \%)$ native valve endocarditis (NVE) and 15 (24.2\%) prosthetic valve endocarditis (PVE), three pacemaker wire (PM) infections and 37\% hospital acquired. Overall, 13/62 (21\%) were caused by MRSA and 76.9 of these were hospital acquired. All the isolates were reported vancomycin and teicoplanin susceptible. Overall, 45 patients $(72.6 \%)$ were cured and 17 (27.4\%) died of endocarditis. Surgery was performed on 20 (32.3\%) and embolic events were documented in 40 cases (64.5\%) (Table 1).

Oxacillin, vancomycin and teicoplanin susceptibility of 62 S.aureus isolates are reported in Table 2. Oxacillin and teicoplanin results in this study were in agreement 
Table 1 Epidemiology of S.aureus endocarditis

\begin{tabular}{|c|c|c|c|c|c|c|c|c|c|c|}
\hline Year & Total & DE (\%) & NVE (\%) & PVE (\%) & PM & MRSA (\%) & HA (\%) & SUR (\%) & EMB (\%) & Cured (\%) \\
\hline$\leq 2000$ & 4 & 4 & 3 & 1 & 0 & 0 & 0 & 2 & 4 & 3 \\
\hline 2001 & 3 & 3 & 3 & 0 & 0 & 1 & 2 & 3 & 2 & 3 \\
\hline 2002 & 1 & 1 & 1 & 0 & 0 & 0 & 0 & 0 & 1 & 1 \\
\hline 2003 & 1 & 1 & 1 & 0 & 0 & 1 & 1 & 0 & 1 & 0 \\
\hline 2004 & 5 & 5 & 4 & 1 & 2 & 0 & 1 & 2 & 1 & 4 \\
\hline 2005 & 14 & 14 & 12 & 2 & 0 & 2 & 5 & 5 & 9 & 11 \\
\hline 2006 & 12 & 11 & 9 & 3 & 1 & 3 & 7 & 6 & 10 & 10 \\
\hline 2007 & 3 & 3 & 2 & 1 & 0 & 0 & 1 & 0 & 2 & 0 \\
\hline 2008 & 5 & 4 & 2 & 3 & 0 & 2 & 2 & 1 & 2 & 4 \\
\hline 2009 & 14 & 11 & 10 & 4 & 0 & 4 & 4 & 1 & 8 & 9 \\
\hline Total & 62 & 57 (91.9) & $47(75.8)$ & $15(24.2)$ & 3 & $13(21.0)$ & $23(37.0)$ & $20(32.3)$ & $40(64.5)$ & $45(72.6)$ \\
\hline
\end{tabular}

DE (definite endocarditis, NVE (native valve endocarditis), PVE (prosthetic valve endocarditis), PM (pace maker), HA (hospital acquired), SUR (surgery), EMB (embolism).

with the laboratory results obtained at admission. Vancomycin susceptibility was not fully confirmed for two isolates tested. One of these was cultured from a patient in Bergamo and one from Perugia; both had macromethod MICs of $4 \mathrm{mg} / \mathrm{l}$ while their MICs with the E-test were $2.5 \mathrm{mg} / \mathrm{l}$ and $2.0 \mathrm{mg} / \mathrm{l}$ respectively.

Methicillin resistance was confirmed in 13 out of 62 (21\%) isolates.

MSSA susceptibility tests: oxacillin geometric mean MIC $0.52 \mathrm{mg} / \mathrm{l}$ (range 0.25-1), $\mathrm{MIC}_{50} 0.35 \mathrm{mg} / \mathrm{l}, \mathrm{MIC}_{90}$ $0.48 \mathrm{mg} / \mathrm{l}$, tolerance rate $63.2 \%$; vancomycin mean MIC $1.69 \mathrm{mg} / \mathrm{l}$ (range 1-2), $\mathrm{MIC}_{50} 1.1 \mathrm{mg} / \mathrm{l}, \mathrm{MIC}_{90} 1.7 \mathrm{mg} / \mathrm{l}$, tolerance rate 18.4\%; teicoplanin mean MIC $1.39 \mathrm{mg} / \mathrm{l}$ (range 1-2), $\mathrm{MIC}_{50} 0.9 \mathrm{mg} / \mathrm{l}, \mathrm{MIC}_{90} 1.7 \mathrm{mg} / \mathrm{l}$, tolerance rate $61.2 \%$.

MRSA susceptibility tests: vancomycin mean MIC $2.3 \mathrm{mg} / \mathrm{l}$ (range 2-4), $\mathrm{MIC}_{50} 1.5 \mathrm{mg} / \mathrm{l}, \mathrm{MIC}_{90} 2.5 \mathrm{mg} / \mathrm{l}$, tolerance rate $30.8 \%$; teicoplanin mean MIC $2.1 \mathrm{mg} / \mathrm{l}$ (range 1-4), $\mathrm{MIC}_{50} 1.3 \mathrm{mg} / \mathrm{l}, \mathrm{MIC}_{90} 2.5 \mathrm{mg} / \mathrm{l}$, tolerance rate $76.9 \%$ (Table 3 ).

Among the 31 oxacillin tolerant strains, 8 (26\%) were also vancomycin tolerant and 18 (58\%) were teicoplanin tolerant. Only one of the oxacillin non tolerant isolates was vancomycin tolerant while $12(66 \%)$ were tolerant to teicoplanin.
Antimicrobial susceptibility tests had been performed at local laboratories at the time of diagnosis, as well, treatment had been decided by the curing physicians on the basis of available susceptibility results and the clinical conditions of patients. MSSA IE antimicrobial therapy consisted of: oxacillin 37, cefazolin 5, vancomycin 4, teicoplanin 1 and not known 2. MRSA IE (13 cases) were treated with vancomycin 10 , teicoplanin 1 and in 2 therapy was not known. Beta-lactam or glycopeptide antimicrobials were administered in combination with rifampin or an aminoglycoside, or a quinolone or a combination of two or more of these antimicrobials for MSSA and MRSA in most of the cases. Need for surgery had been individualised following international indications. Overall, the cure rate was $79.6 \%$ for MSSA: 31 oxacillin, 4 cefazolin, 3 vancomycin and 1 teicoplanin while the cure rate for MRSA was 46.2\%: 5 vancomycin and 1 unreported treatment $(\mathrm{p}<0.032)$. There was no evidence of any differences with regard to need for surgery or embolic events between MSSA and MRSA IE (Table 4). There were only four patients with MRSA IE vancomycin tolerant and all of these had been treated with antimicrobial combinations including vancomycin and had a cure rate of $25 \%$ (Table 4 ).

Table 2 Bacteriostatic activity of oxacillin and glycopeptides

\begin{tabular}{|c|c|c|}
\hline Antibiotics & Macromethod MICs N.susceptible/N.tested (\%) & E-test MICs N.susceptible/N.tested (\%) \\
\hline 口Oxacillin (MIC: $\mathrm{S} \leq 2 \mathrm{mg} / \mathrm{l})(\mathrm{DISK}: \mathrm{S} \geq 22 \mathrm{~mm})$ & $49 / 62(79.0 \%)$ & $49 / 62(79.0 \%)$ \\
\hline */**Vancomycin $(\mathrm{S} \leq 2 \mathrm{mg} / \mathrm{l})$ & $60 / 62(96.7 \%)$ & $61 / 62(98.3 \%)$ \\
\hline *Teicoplanin $(\mathrm{S} \leq 8 \mathrm{mg} / \mathrm{l})$ & $62 / 62(100 \%)$ & $62 / 62(100 \%)$ \\
\hline **Teicoplanin ( $\mathrm{S} \leq 2 \mathrm{mg} / \mathrm{l})$ & $60 / 62(96.7 \%)$ & $60 / 62(96.7 \%)$ \\
\hline
\end{tabular}

$\square$ CLSI and EUCAST susceptibility break point, cefoxitin disk diffusion same results as oxacillin 
Table 3 MIC geometric mean, $\mathrm{MIC}_{50}, \mathrm{MIC}_{90}$ and rate of tolerance (macromethod)

\begin{tabular}{|c|c|c|c|c|c|c|c|c|c|c|c|c|}
\hline & Oxacillin & & & & Vancomycin & & & & Teicoplanin & & & \\
\hline & $\begin{array}{l}\text { Mean MIC (mg/l) } \\
\text { (range) }\end{array}$ & $\begin{array}{l}\mathrm{MIC}_{50} \\
(\mathrm{mg} / \mathrm{l})\end{array}$ & $\begin{array}{l}\mathrm{MIC}_{90} \\
(\mathrm{mg} / \mathrm{l})\end{array}$ & $\begin{array}{l}\text { N.tolerant } \\
\text { (\%) }\end{array}$ & $\begin{array}{l}\text { Mean MIC (mg/l) } \\
\text { (range) }\end{array}$ & $\begin{array}{l}\mathrm{MIC}_{50} \\
(\mathrm{mg} / \mathrm{l})\end{array}$ & $\begin{array}{l}\mathrm{MIC}_{90} \\
(\mathrm{mg} / \mathrm{l})\end{array}$ & $\begin{array}{l}\text { N. tolerant } \\
\text { (\%) }\end{array}$ & $\begin{array}{l}\text { Mean MIC (mg/l) } \\
\text { (range) }\end{array}$ & $\begin{array}{l}\mathrm{MIC}_{50} \\
(\mathrm{mg} / \mathrm{l})\end{array}$ & $\begin{array}{l}\mathrm{MIC}_{90} \\
(\mathrm{mg} / \mathrm{l})\end{array}$ & $\begin{array}{l}\text { N. tolerant } \\
(\%)\end{array}$ \\
\hline $\begin{array}{l}\text { MSSA N.49 } \\
(79 \%)\end{array}$ & $0.52(0.25-1)$ & 0.35 & 0.48 & $\begin{array}{l}31 / 49 \\
(63.2 \%)\end{array}$ & $1.69(1-2)$ & 1.1 & 1.7 & 9/49 (18.4\%) & $1.39(1-2)$ & 0.9 & 1.7 & $\begin{array}{l}30 / 49 \\
(61.2 \%)\end{array}$ \\
\hline $\begin{array}{l}\text { MRSA N.13 } \\
(21 \%)\end{array}$ & NA & NA & NA & NA & $2.3(2-4)$ & 1.5 & 2.5 & $4 / 13(30.8 \%)$ & $2.1(1-4)$ & 1.3 & 2.5 & $\begin{array}{l}10 / 13 \\
(76.9 \%)\end{array}$ \\
\hline
\end{tabular}


Table 4 Rates of cure, surgery, and embolism in endocarditis caused by MSSA and MRSA

\begin{tabular}{llll}
\hline & Cured N. (\%) & Surgery N. (\%) & Embolic events N. (\%) \\
\hline MSSA N. $49(79 \%)$ & $39 / 49 *(79.6 \%)$ & $16 / 49 * *(32.7 \%)$ & $33 / 499^{* * *}(67.3 \%)$ \\
\hline MRSA N. $13(21 \%)$ & $6 / 13^{*}(46.2 \%)$ & $4 / 13^{* *}(30.8 \%)$ & $7 / 13^{* * *}(53.8 \%)$ \\
\hline Vancomycin tolerant N. 4/13 (30.8\%) & $1 / 4^{\circ}(25,0 \%)$ & & \\
Vancomycin non tolerant N. $9 / 13(69.2 \%)$ & $5 / 99^{\circ}(55,5 \%)$ & & \\
\hline${ }^{*} \mathrm{p}<0.032,{ }^{* *} \mathrm{p}=1,{ }^{* * *} \mathrm{p}=0.51,{ }^{\circ} \mathrm{p}=0.55$. & & &
\end{tabular}

A negative effect of oxacillin tolerance was not observed either in the entire subgroup of IE cases caused by MSSA or in the subgroup of patients with MSSA IE being treated with an antibiotic regimen containing oxacillin (Table 5).

\section{Discussion}

This study was designed to assess the rate of tolerance to oxacillin, vancomycin and teicoplanin among S.aureus isolates in patients with IE. Thereafter, we sought to determine a relationship between in vitro bactericidal tests and clinical outcome.

The bactericidal test results of this study, defined by MBCs, showed that vancomycin bactericidal activity was not inferior to that of oxacillin and teicoplanin against MSSA. Glycopeptide tolerance was more common among MSSA oxacillin tolerant strains but a few non tolerant MSSA oxacillin with high MBC for glycopeptides were also observed.

Among MRSA isolates, vancomycin tolerance rate was inferior to that of teicoplanin and all the 4 vancomycin tolerant isolates were also teicoplanin tolerant.

Previous papers have reported that vancomycin has in vitro bactericidal activity slower than that of nafcillin with more frequent clinical failures in animal models and also patients being treated with vancomycin [4-6]. Technical variables such as inoculum size, growth conditions and killing curves, instead of MBCs, may be responsible for some of the differences in the results of this study. May et al. [20] found MBCs and the killing rates comparable with some discrepancies. Lack of killing in vitro has also been hypothesised to be a reversible phenotypic response due to growth conditions of the test with some types of constitutional changes noted in tolerant strains [21,22]. It has been reported that most beta-lactam tolerant strains of S.aureus show cross tolerance to vancomycin and teicoplanin [23].

Regarding clinical outcome, $79 \%$ of IE cases in this study were caused by MSSA. In these patients, oxacillin was the most common antibiotic prescribed and it was usually given in combination with other antibiotics in both oxacillin tolerant and oxacillin non-tolerant MSSA infections. Analysis was unable to demonstrate either increased mortality or morbidity in oxacillin tolerant MSSA IE patients. Strikingly, a non-statistically significant higher cure rate was observed in patients with IE caused by oxacillin tolerant MSSA in both the entire MSSA IE group and in the MSSA subgroup treated with oxacillin.

Despite its greater in vitro bactericidal activity, a higher rate of clinical failures was observed among patients with MRSA IE. Among MRSA IE patients, higher rates of clinical failure were observed among patients with endocarditis caused by vancomycin tolerant MRSA strains, but there were only four patients in this group of patients to comment on.

With regard to bacteriostatic activity, methicillin resistance was confirmed in $21 \%$ of the isolates. Methicillin resistance occurred more commonly among hospital acquired infections with the exception of three cases. One of these should be classified as health care associated even though the isolate had a mecA cassette type $\mathrm{V}$, which is a marker for methicillin-resistant community acquired infection [24] and was susceptible to all other classes of antibiotics except penicillin. This patient reported a traumatic tibia fracture, was treated with external devices and discharged from hospital. Subsequently, the patient developed osteomyelitis and

Table 5 Rates of cure, surgery, and embolism in MSSA endocarditis caused by oxacillin tolerant and oxacillin nontolerant strains

\begin{tabular}{|c|c|c|c|}
\hline MSSA 49/62 (79\%) & Cured N. (\%) & Surgery N. (\%) & Embolic events N. (\%) \\
\hline Oxacillin tolerant N. 31 (63.2\%) & $26 / 31 *(83.9 \%)$ & $10 / 31 * *(32.3 \%)$ & $21 / 31 * *(67.7 \%)$ \\
\hline Oxacillin non tolerant N. 18 (36.8\%) & $13 / 18 *(72.2 \%)$ & $6 / 18 * *(33.3 \%)$ & $12 / 18 * *(66.7 \%)$ \\
\hline $\begin{array}{l}\text { MSSA oxacillin tolerant treated with oxacillin/total MSSA oxacillin tolerant N. } \\
24 / 31(77.4 \%)\end{array}$ & $21 / 24^{\circ}(87.5 \%)$ & - & - \\
\hline $\begin{array}{l}\text { MSSA oxacillin non tolerant treated with oxacillin/total MSSA oxacillin non- } \\
\text { tolerant N. } 13 / 18(72.2 \%)\end{array}$ & $10 / 13^{\circ}(76.9 \%)$ & - & - \\
\hline
\end{tabular}


endocarditis with multiple septic lung emboli and myocardial abscess. The other two cases were community acquired MRSA IE from the Bergamo cohort.

Concordant susceptibility test results were obtained also with teicoplanin.

With regard to vancomycin, there was no full agreement on the grade of susceptibility obtained at admission. Two isolates had vancomycin macro-method MICs of $4 \mathrm{mg} / \mathrm{l}$, which are intermediate susceptible values. The obtained values were still in the susceptible range when performing the E-test, being $2 \mathrm{mg} / \mathrm{l}$ for the Perugia isolate and $2.5 \mathrm{mg} / \mathrm{l}$ for the Bergamo isolate. The former was methicillin resistant, hospital acquired, non tolerant to vancomycin but teicoplanin tolerant, cultured in early prosthetic infection with valve abscess. The patient treatment included teicoplanin, followed by daptomycin and rifampin then tigecycline. The patient died. The latter isolate was methicillin resistant, hospital acquired and vancomycin tolerant, cultured from a case of pace-maker infection. The patient underwent both the surgical removal of the device and vancomycin plus fosfomicin and co-trimoxazole treatment with cure.

In this study, all 62 isolates had slightly higher macromethod vancomycin MICs than with the E-test. Previously in literature, higher vancomycin MICs have been observed with the E-test compared to the micro-dilution method. Nonetheless, the micro-dilution method and Etest both have been reported to perform differently than disk diffusion and some automated systems $[25,26]$. Given this, while these in vitro vancomycin susceptibility testing parameters are being standardised, it may be advisable to assess vancomycin MICs with more than one method and to carry out a close clinical and microbiological follow up while the patients are being treated with vancomycin.

A progressive increase in vancomycin MICs over time was not observed in this study $[7,25,26]$ however, there were very few strains included before the year 2004 and all of these, though methicillin susceptible, already had vancomycin MIC of 1-2 mg/l.

\section{Conclusions}

This study reports that oxacillin and teicoplanin tolerance was common among S.aureus and more common than vancomycin. This study was unable to show a statistically significant correlation between bactericidal activity in vitro and clinical outcome. However, one must consider that these results were from a retrospective analysis and treatment was not standardized. Additional, prospective standardized studies are needed to evaluate if in vitro bactericidal tests are valid predictors of clinical outcome in staphylococcal endocarditis.

\section{Acknowledgements}

We would like kindly thank Professor Stefania Stefani, Department of Microbiology, University of Catania, Catania, Italy for having examined the staphylococcal mecA cassette in the isolate from Perugia.

\section{Author details}

'Section of Infectious Diseases, Department of Experimental Medicine and Biochemical Sciences, University of Perugia, Perugia, Italy. ${ }^{2}$ Infectious Disease Department, Ospedali Riuniti di Bergamo, Bergamo, Italy. ${ }^{3}$ Unit of Microbiology and Virology, Ospedali Riuniti di Bergamo, Bergamo, Italy.

\section{Authors' contributions}

PMB designed the study, supervised laboratory experiments, performed clinical examinations, recruited patients, analysed the data and drafted the manuscript. AM and AC collected bacterial isolates, performed on admission susceptibility tests and laboratory experiments. VR, LS, AS and CP carried out clinical examinations and the recruitment of patients. AR and AG collected strains and performed on admission susceptibility tests. AS and RP analysed the data. GS and FB revised the manuscript. All authors read and approved the final manuscript.

\section{Competing interests}

The authors declare that they have no competing interests.

Received: 26 January 2011 Accepted: 9 June 2011

Published: 9 June 2011

\section{References}

1. Fowler VG, Miro JM, Hoen BH, Abrutyn E, Rubinstein E, Corey GR, Spelman D, Bradley SF, Barsic B, Pappas PA, Anstrom KJ, Wray D, Fortes CQ, Anguera I, Athan E, Jones P, van der Meer JT, Elliot TS, Levine DP, Bayer AS, ICE Investigators: Staphylococcus aureus endocarditis: a consequence of medical progress. JAMA 2005, 24:3012-3021.

2. Hill EE, Herijgers P, Herregods M-C, Peetermans WE: Evolving trends in infective endocarditis. Clinic Microbiol Infect 2006, 12(1):5-12

3. Naimi TS, LeDell KH, Como-Sabetti K, Borchardt SM, Boxrud DJ, Etienne J, Johnson SK, Vandenesch F, Fridkin S, O'Boyle C, Danila RN, Lynfield R: Comparison of community and health care-associated methicillinresistant Staphylococcus aureus infections. JAMA 2003, 290(22):2976-2984.

4. Sakoulas G, Moise-Broder PA, Schentag J, Forrest A, Moellering RC Jr, Eliopoulis GM: Relationship of MIC and bactericidal activity to efficacy of vancomycin for treatment of methicillin-resistant Staphylococcus aureus bacteremia. J Clin Microbiol 2004, 42(6):2398-2402.

5. Cantoni L, Glauser MP, Bille J: Comparative efficacy of daptomycin, vancomycin and cloxacillin for the treatment of Staphylococcus aureus endocarditis in rats and role of test conditions in this determination. Antimicrob Agents Chemother 1990, 34(12):2348-2353.

6. Small PM, Chambers HF: Vancomycin for Staphylococcus aureus endocarditis in intravenous drug users. Antimicrob Agents Chemother 1990, 34(6):1227-1231.

7. Steinkraus $G$, White $R$, Friedrich $L$ : Vancomycin MIC creep in nonvancomycin-intermediate Staphylococcus aureus (VISA), vancomycin susceptible clinical methicillin-resistant S.aureus (MRSA) blood isolates from 2001-2005. J Antimicrob Chemother 2007, 60:788-794.

8. Monaco M, Sanchini A, Grudmann H: Vancomycin-heteroresistant phenotype in invasive methicillin-resistant Staphylococcus aureus isolates belonging to spa type 041. Eur J Clin Microbiol Infect Dis 2010, 29:771-777.

9. Charles PGP, Ward PB, Johnson DR, Benjamin PH, Grayson L: Clinical features associated with bacteremia due to heterogeneous vancomycinintermediate Staphylococcus aureus. Clin Infect Dis 2004, 38:448-451.

10. Sakoulas G, Moellering RC Jr, Eliooulos GM: Adaptation of methicillinresistant Staphylococcus aureus in the face of vancomycin therapy. Clin Infec Dis 2006, 42(suppl):40-50.

11. Traczewski MM, Bradley DK, Steenbergen JN, Brown SD: Inhibitory and bactericidal activity of daptomycin, vancomycin and teicoplanin against methicillin-resistant Staphylococcus aureus isolates collected from 1985 to 2007. Antimicrob Agents Chemoter 2009, 53(9):1735-1738.

12. Denny AE, Peterson R, Gerding DN, Hall WH: Serious staphylococcal infections with strains tolerant to bactericidal antibiotics. Arch Intern Med 1979, 139:1026-1031 
13. Rajashekaraiah KR, Rice T, Rao VS, Marsh D, Ramakrisna B, Kallick CA: Clinical significance of tolerant strains of Staphylococcus aureus in patients with endocarditis. Ann Intern Med 1980, 93(6):796-801.

14. Goldman PL, Petersdorf RG: Significance of methicillin tolerance in experimental staphylococcal endocarditis. Antimicrob Agents Chemoter 1979, 15(6):802-806.

15. French GL: Bactericidal agents in the treatment of MRSA infections-the potential role of daptomycin. J Antimicrob Chemother 2006, 58:1107-1117.

16. Clinical and Laboratory Standard Institute: Performance Standards for Antimicrobial Susceptibility Testing; 18th Informational Supplement. M100-S18. Clinical and Laboratory Standard Institute, Wayne, PA; 2008.

17. Clinical and Laboratory Standard Institute: Methods for Dilution Antimicrobial Susceptibility Tests for Bacteria That Grow Aerobically; Approved Standard. Clinical and Laboratory Standard Institute, Wayne, PA, 8 2009, M07-A8.

18. Clinical and Laboratory Standard Institute: Methods for Determining Bactericidal Activity of Antimicrobial Agents; Approved Guideline. M26A. 1999. Clinical and Laboratory Standard Institute, Wayne, PA.

19. Miller JM: Calculating MIC50. J Antimicrob Chemother 1991, 27(6):863-864.

20. May J, Shannon K, King A, French G: Glycopeptide tolerance in Staphylococcus aureus. J Antimicrob Chemother 1988, 42:189-197.

21. Sabath LD, Lavadiere M, Wheeler N, Blazevic D, Wilkinson BJ: A new type of penicillin resistance in Staphylococcus aureus. Lancet i 1997, 443-447.

22. Handwerger S, Tomasz A: Antibiotic tolerance among clinical isolates of bacteria. Rev Infect Dis 1985, 7:368-386.

23. Perry JD, Jones AL, Gould F: Glycopeptide tolerance in bacteria causing endocarditis. J Antimicrob Chemother 1999, 44:121-124.

24. Chen L, Mediavilla JR, Oliveira DC, Willey BM, de Lencastre H, Kreiswirth BN: Multiplex Real-Time PCR for rapid staphylococcal cassette chromosome mec typing. J Clin Microbiol 2009, 47(11):3692-3706.

25. Jones RN: Microbiological features of vancomycin in the $21^{\text {st }}$ century: minimum inhibitory concentration creep, bactericidal/static activity and applied breakpoints to predict clinical outcomes or detect resistant strains. Clin Infect Dis 2006, 42(suppl):13-24.

26. Sader HS, Rhomberg PR, Jones RN: Nine-Hospital study comparing broth microdilution and E-test method results for vancomycin and daptomycin against methicillin-resistant Staphylococcus aureus. Antimicrob Agents Chemother 2009, 53(7):3162-3165.

doi:10.1186/1476-0711-10-26

Cite this article as: Pasticci et al:: Bactericidal activity of oxacillin and glycopeptides against Staphylococcus aureus in patients with endocarditis: Looking for a relationship between tolerance and outcome. Annals of Clinical Microbiology and Antimicrobials 2011 10:26.

\section{Submit your next manuscript to BioMed Central and take full advantage of:}

- Convenient online submission

- Thorough peer review

- No space constraints or color figure charges

- Immediate publication on acceptance

- Inclusion in PubMed, CAS, Scopus and Google Scholar

- Research which is freely available for redistribution

Submit your manuscript at www.biomedcentral.com/submit
Biomed Central 